\title{
Pharmacogenetic Study on Risperidone Long-Acting Injection
}

\author{
Influence of Cytochrome P450 2D6 and Pregnane X Receptor on \\ Risperidone Exposure and Drug-Induced Side-Effects
}

\author{
Eva Choong, PhD, * Andrea Polari, MD, † Rigobert Hervais Kamdem, MD, $\neq$ Nicola Gervasoni, MD, $\S$ \\ Caesar Spisla, MD,// Eveline Jaquenoud Sirot, PhD,I Graziella Giacometti Bickel, MD, $\neq$ \\ Guido Bondolfi, MD, $\S$ Philippe Conus, MD, † and Chin B. Eap, PhD*\#
}

\begin{abstract}
Risperidone is metabolized by polymorphic enzymes, and a large variability in plasma concentration and therapeutic response is observed. Risperidone long-acting injection (RLAI) avoids the first-pass effect, and little is known about the influence of gene polymorphisms involved in its pharmacokinetics. The influence on plasma concentrations of risperidone (RIS), its metabolite 9-hydroxy-risperidone, and on adverse effects were investigated for polymorphisms of cytochrome P450 2D6 (CYP2D6) (*3, *4, *5, *6), CYP3A (CYP3A4*1B, CYP3A4 rs 4646437, CYP $3 A 5 * 3, C Y P 3 A 7 * 1 C), A B C B 1(1236 C>T, 2677 G>T$, $3435 C>T$ ), NR1/2 coding for pregnane $\mathrm{X}$ receptor (rs1523130, rs 2472677, rs7643645), and for CYP3A activity measured by a phenotyping test. Forty-two patients with at least 4 consecutive unchanged doses of RLAI were included in a multicenter cross-sectional study. A $55 \%$ lower dose-adjusted plasma levels of RIS were observed for CYP2D6 ultrarapid metabolizers $(\mathrm{n}=5)$ as compared with CYP2D6 intermediate metabolizers $(P<0.007)$. NR1/2 polymorphism $(r s 7643645 A>G)$ influenced RIS exposure with a 2.8 -fold lower active moiety $(P=0.031)$ in GG compared with the AA genotype. This was confirmed in a second independent cohort $(\mathrm{n}=16)$. Furthermore, high-density lipoprotein cholesterol was positively correlated with CYP3A activity $(P=0.01)$, and the NRI/2 (rs2472677) polymorphism was associated with different adverse effects including prolactin plasma levels adjusted for age and sex. In conclusion, our results confirmed the influence of CYP2D6 genotype on plasma levels of RIS. This is the first report on the influence of NRI/2 polymorphisms on RLAI exposure and on drug-induced adverse effects. These results should be validated in larger cohorts.
\end{abstract}

Key Words: risperidone, depot formulation, CYP2D6, NR1/2, $\mathrm{ABCB} 1, \mathrm{CYP} 3 \mathrm{~A}$

(J Clin Psychopharmacol 2013;33: 289-298)

From the *Unit of Pharmacogenetics and Clinical Psychopharmacology, Centre for Psychiatric Neuroscience, Department of Psychiatry, University Hospital Centre and University of Lausanne; and †Service de Psychiatrie Générale, Département de Psychiatrie-CHUV, Hôpital de Cery, Prilly; \$Fribourg Mental Health Network, Fribourg; §Department of Mental Health and Psychiatry, University Hospital of Geneva, Geneva; \#Psychiatrische Dienste Aargau AG, Aarau; $\uparrow$ Psychiatrische Dienste Aargau AG, mediQ, Klinik Königsfelden, Brugg; and \#School of Pharmaceutical Sciences, University of Geneva, University of Lausanne, Geneva, Switzerland. Received September 15, 2011; accepted after revision October 24, 2012.

Reprints: Chin B. Eap, PhD, Hôpital de Cery, 1008 Prilly, Lausanne, Switzerland (e-mail: chin.eap@chuv.ch).

This work has been funded in part by the Swiss National Research

Foundation (no. 320030-120686) and by a nonrestricted educational grant by Janssen-Cilag Switzerland. The funding sources had no role in the writing of the manuscript or in the decision to submit it for publication. Copyright (C) 2013 by Lippincott Williams \& Wilkins

ISSN: 0271-0749

DOI: $10.1097 / J C P .0 b 013 \mathrm{e} 31828 \mathrm{f} 62 \mathrm{~cd}$
$\mathrm{T}^{\mathrm{h}}$ he atypical antipsychotic risperidone (RIS) is metabolized in the organism into its main metabolite 9-hydroxyrisperidone (OHRIS), both substances contributing to the pharmacological activity, their sum being called "active moiety." Risperidone produces a relatively low incidence of extrapyramidal symptoms (EPSs) at low doses. However, a relationship between occurrence of parkinsonian adverse effects and plasma levels of RIS and OHRIS has been reported, ${ }^{1}$ and RIS produces dose-related increases in plasma prolactin levels both in men and women. ${ }^{2}$

Several in vitro and in vivo studies showed that the enzyme cytochrome P450 2D6 (CYP2D6) and, to a lesser extent, CYP3A play a major role in the metabolism of RIS to OHRIS., ${ }^{3,4}$ Thus, concomitant administration of CYP2D6 inhibitors such as paroxetine or fluoxetine significantly increases RIS plasma concentration. ${ }^{3,4}$ Such an increase of the RIS/OHRIS ratio in plasma following the administration of CYP2D6 inhibitor does not necessarily result in an increase of adverse effects ${ }^{3}$ but could be a risk factor, as suggested by the appearance of parkinsonian symptoms in 1 patient 2 weeks after the introduction of $20 \mathrm{mg} / \mathrm{d}$ of paroxetine and an important increase of the active moiety plasma levels. ${ }^{4}$ In vivo, the CYP3A inhibitor itraconazole increases, whereas the CYP3A inducers carbamazepine and rifampicin reduce the plasma concentrations of RIS, OHRIS, and of the active moiety. ${ }^{5-7}$

Accordingly, several studies have shown that the polymorphism of CYP2D6 gene, leading to different metabolism activity, significantly contributes to the wide interindividual variability of RIS and OHRIS plasma concentrations on the same oral dose. ${ }^{8,9}$ With regard to the metabolism by CYP3A, a term that in humans reflects the collective activity of CYP3A4, CYP3A5, and CYP3A7, the large interindividual variability of these isoforms is poorly determined by genotyping procedures and is presently better assessed by a phenotyping test using, for example, midazolam as probe. ${ }^{10}$ To our knowledge, no study has ever attempted to determine if, and to what extent, the interindividual variability of CYP3A activity as measured by a phenotyping test, could contribute to the interindividual variability of RIS and OHRIS plasma levels.

Besides CYP2D6 and CYP3A, RIS is a substrate of the drug efflux transporter P-glycoprotein (P-gp) encoded by the $A B C B 1$ gene. It has been shown that the single-nucleotide polymorphisms (SNPs) of $A B C B 1$ (C3435T and G2677T/A) do not influence the steady-state plasma concentrations of RIS and OHRIS after the oral intake of $3 \mathrm{mg} / \mathrm{d}$ of RIS. ${ }^{11}$ However, as P-gp is expressed at the blood-brain barrier, it has been suggested that this protein plays a much more important role in modulating drug concentrations in the brain than in the plasma. ${ }^{12}$ 
In addition, attention has been recently drawn to modulators of gene expression in phases I and II metabolism and in drug transport. Pregnane $\mathrm{X}$ receptor (PxR) encoded by $N R 1 / 2$ gene mediates induction of several drug detoxification genes including $C Y P 3 A 4 / 5$ and $A B C B 1 .^{13,14} N R 1 / 2$ mRNA is predominantly expressed in the intestine, liver, and kidney, and $N R 1 / 2$ is activated by many endogenous and exogenous ligands. ${ }^{13} N R 1 / 2$ SNPs in promoter and intron 1 have been associated with alteration of CYP3A activity in vitro, the most consistent results being found for SNPs rs1523130, rs2472677, and $r_{s} 7643645 .^{15}$

Risperidone is also available as a long-acting depot formulation (Risperdal Consta; Janssen-Cilag, Baar, Switzerland), with a recommended injection every 2 weeks. The long-acting depot preparations bypass the deactivating process of the intestines and of the liver, which has been reported to lead to more predictable and stable drug plasma concentration. ${ }^{16}$ There is limited information on influence of genetic factors on RIS long-acting injectable (RLAI) form as well as on its pharmacokinetics. One study showed a lower active moiety level in a patient taking RLAI and carbamazepine, a CYP3A inducer, compared with other patients receiving the same RLAI dose. ${ }^{17}$ In addition, we reported a case of rapid RIS elimination in 1 patient necessitating a very high dosage of RLAI ( $125 \mathrm{mg} / 2$ weeks) before obtaining a substantial improvement of his clinical state. ${ }^{18}$ Moreover, it is possible that changing from oral to RLAI may be associated with higher RIS/OHRIS ratios, which might be a clinically relevant issue. ${ }^{19}$ This highlights the need for a better understanding of the in vivo influence of genetic and environmental factors on RIS and OHRIS plasma levels after injection of RIS depot.

The major aim of this study was to analyze the influence of gene polymorphisms linked to RIS metabolism and transport (genotyping of $C Y P 2 D 6, C Y P 3 A, A B C B 1, N R 1 / 2$, and phenotyping of CYP3A) in a group of patients receiving RLAI in steady-state conditions.

\section{MATERIALS AND METHODS}

\section{Subjects and Study Design}

All the patients between 18 and 65 years old presenting with a psychotic disorder based on International Classification of Diseases, 10th Revision criteria and receiving RLAI were screened from February 2007 to December 2009. Because pregnancy was an exclusion criterion, a pregnancy test was performed at day 0 before inclusion of women of childbearing age. No change in the dosage of the comedications was allowed for at least 1 week before inclusion in the study ( 2 months for fluoxetine), and RLAI has been administered for at least 2 months (4 injections) at constant dose. At inclusion, patients had no history of substance dependence, organic psychiatric illness, or uncontrolled medical illness and were not taking oral RIS. Complete inclusion comprised a visit on the day of RIS depot injection (day 0) and 7 days later (day 7). No fasting period was required.

This naturalistic cross-sectional study was conducted in 4 psychiatric centers in Switzerland (Lausanne, Geneva, Königsfelden, Marsens) and was approved by the respective ethics committees as well as by the Swiss Agency for Therapeutic Products (Swissmedic). The study was performed according to the Good Clinical Practices, and written informed consent was obtained from all patients.

Patients with RLAI plasma levels from 2 independent clinical studies, which were approved by their respective ethics committee (of Geneva and Lausanne University hospitals), were evaluated to replicate the positive findings found for the $N R 1 / 2$ gene (called hereafter replication group). Written informed consent was given by all subjects. Blood samplings were performed at the end of the dosing interval (ie, 14 days after the injection) \pm 3 days ( 3 patients at day 11,1 at day 12,1 at day 13 , 11 at day 14).

\section{Measurements}

Somatic disorders, comedications, and clinical variables were recorded from medical files. Subjects were asked to report any change in their medication or lifestyle during the study. The year of onset of psychiatric disorder was found in the medical files; otherwise, an estimation was given by the patients. An evaluation of the global illness severity was performed using the Clinical Global Impression Scale (CGI Scale from 1 to 7 , with 7 being the most severe presentation). ${ }^{20} \mathrm{Ex}$ trapyramidal adverse effects were assessed by the SimpsonAngus Scale (SAS), scored from 0 to 40, with an SAS score below 3 being reported as normal. ${ }^{21}$ Furthermore, spontaneous self-reported adverse events and adverse effects were also collected. All investigators involved in the rating scales assessments were trained before recruitment to minimize interrater variability.

Risperidone and OHRIS plasma concentrations, CYP3A activity, and prolactin levels were measured at days 0 and 7 , whereas total cholesterol (CHOLtot) and high-density lipoprotein (HDL) cholesterol were measured at day 0. Quantification of RIS and OHRIS plasma levels was performed using highperformance liquid chromatography-mass spectrometry in the positive ESI mode (Agilent Series 1100 MSD Single Quadrupole; Agilent Technologies, Geneva, Switzerland; unpublished method, detailed method available on request). Briefly, a liquidliquid extraction step was performed before injecting the extract into a C18 Nucleosil column (EC 125/2 Nucleosil 100-5; Macherey-Nagel, Oensingen, Switzerland) using a mobile phase containing $35 \%$ tetrahydrofuran, $65 \%$ ammonium nitrate $4 \mathrm{mM}$, and $1.5 \%$ methanol. Intraday and interday coefficients of variation were between $1 \%$ and $3 \%$ for the determination of RIS and OHRIS. The low limit of quantification was 0.1 and $0.2 \mathrm{ng} / \mathrm{mL}$ for RIS and OHRIS, respectively. External quality controls (LGC Standards Proficiency Testing, Lancashire, UK) were successfully quantified since the analytical procedure validation. The active moiety and the RIS/OHRIS ratio were calculated for days 0 and 7 .

Total cholesterol and HDL cholesterol levels were quantified by a Cobas Integra 400 (Roche Diagnostic, Basel, Switzerland). Prolactin levels were quantified by immunoassay on an Abbott Axsym system (Abbott, Wiesbaden, Germany). High CHOLtot levels were defined as $6.2 \mathrm{mmol} / \mathrm{L}$ or greater, and normal HDL levels as $1.3 \mathrm{mmol} / \mathrm{L}$ or greater for women and as $1.0 \mathrm{mmol} / \mathrm{L}$ or greater for men. ${ }^{22}$ Dyslipidemia was defined as a concentration of CHOLtot and/or HDL outside the limits, and/or when cholesterol-lowering drug is prescribed. High level of prolactin was defined as $50 \mathrm{ng} / \mathrm{mL}$ or greater for women and as $40 \mathrm{ng} / \mathrm{mL}$ or greater for men, which represents twice the threshold for normal values. ${ }^{23}$ Body mass index (BMI) is defined as body weight $/$ height $^{2}\left(\mathrm{~kg} / \mathrm{m}^{2}\right)$, and BMI of $30 \mathrm{~kg} / \mathrm{m}^{2}$ or greater is considered as obese. An increase of at least $10 \%$ of the baseline weight was considered as a relevant weight gain.

For CYP3A phenotyping analyses, an oral microdose $(75 \mu \mathrm{g})$ of midazolam was given 30 minutes before the blood sample collection. The midazolam, and its metabolite, 1-hydroxymidazolam, plasma levels were measured by gas chromatography-mass spectrometry, as described previously. ${ }^{24}$ The 1-hydroxymidazolam/ midazolam ratio (MR), a marker of CYP3A activity, ${ }^{10,24}$ was 
TABLE 1. Clinical Characteristics of the Patients and of the Replication Group

\begin{tabular}{|c|c|c|c|}
\hline Variables & & Patients & Replication Group \\
\hline $\mathrm{n}$ & & 42 & 16 \\
\hline Sex, male & $\mathrm{n}(\%)$ & $30(71)$ & $13(81)$ \\
\hline Age, y & Median (range) & $35(18-63)$ & $36(21-63)$ \\
\hline \multicolumn{4}{|l|}{ Ethnic group } \\
\hline Caucasian & n $(\%)$ & $32(76)$ & $10(62)$ \\
\hline African & n $(\%)$ & $7(17)$ & $3(19)$ \\
\hline Other groups & n $(\%)$ & $3(7)$ & $3(19)$ \\
\hline \multicolumn{4}{|l|}{ Psychiatric diagnosis $(I C D-10)$} \\
\hline Schizoaffective disorder (F25) & n $(\%)$ & $8(19)$ & - \\
\hline Schizophrenia (F20) & n $(\%)$ & $34(81)$ & - \\
\hline Psychiatric disorder duration, ${ }^{*} \mathrm{y}$ & Median (range) & $9(1-42)$ & - \\
\hline Prolactin, $\mathrm{ng} / \mathrm{mL}$ & Median (range) & $33(11-271)$ & - \\
\hline High prolactin $\dagger$ & $\mathrm{n}(\%)$ & $17(40)$ & - \\
\hline \multicolumn{4}{|l|}{ BMI, $\mathrm{kg} / \mathrm{m}^{2}$} \\
\hline Initial BMI & Median (range) & $24.8(16.9-36.3)$ & - \\
\hline Current BMI & Median (range) & $27.1(18.0-39.9)$ & $27.8(23.5-33.6)$ \\
\hline \multicolumn{4}{|l|}{ Cardiovascular risks§ } \\
\hline Initial prevalence of obesity & $\mathrm{n}(\%)$ & $9(23)$ & - \\
\hline Current prevalence of obesity & $\mathrm{n}(\%)$ & $11(26)$ & $4(27)$ \\
\hline Smoker & n $(\%)$ & $34(81)$ & $11(69)$ \\
\hline CHOLtot, $\mathrm{mmol} / \mathrm{L}$ & Median (range) & $4.8(1.2-9.6)$ & - \\
\hline High CHOLtot & $\mathrm{n}(\%)$ & $6(14)$ & - \\
\hline HDL cholesterol, mmol/L & Median (range) & $1.2(0.6-2.4)$ & - \\
\hline Low HDL cholesterol & n $(\%)$ & $14(33)$ & - \\
\hline RLAI duration, mo & Median (range) & $8.5(2-78)$ & $12(3-45)$ \\
\hline RLAI dose, $\mathrm{mg} / 2 \mathrm{wk}$ & Median (range) & $37.5(25-75)$ & $38(25-100)$ \\
\hline
\end{tabular}

Note that BMI data are available for $\mathrm{n}=15$ in replication group.

*Time duration from the first psychiatric episode to the inclusion in the study.

$\dagger$ High prolactin is considered as prolactin of greater than $50 \mathrm{ng} / \mathrm{mL}$ for women and greater than $40 \mathrm{ng} / \mathrm{mL}$ for men.

\$Before RLAI treatment initiation (data available for $\mathrm{n}=39$ ). All variables without $\$$ indicated data at inclusion in the study.

§Patient is considered as obese when BMI if $30 \mathrm{~kg} / \mathrm{m}^{2}$ or greater; CHOLtot $6.2 \mathrm{mmol} / \mathrm{L}$ or greater and HDL cholesterol less than $1 \mathrm{mmol} / \mathrm{L}$ for men and les than $1.3 \mathrm{mmol} / \mathrm{L}$ for women are considered as cardiovascular risk factors.

ICD-10 indicates International Classification of Diseases, 10th Revision.

calculated for all subjects. A weak CYP3A activity was defined as MR of less than 1.9, and an extensive activity as MR of 1.9 or greater. $^{25}$

Genomic DNA was extracted from the EDTA blood samples using the FlexiGene DNA Kit (Qiagen, Hombrechtikon, Switzerland). Single-nucleotide polymorphisms selected from literature were analyzed by real-time polymerase chain reaction (PCR) with the 5'-nuclease allelic discrimination assays according to previous studies or according to the manufacturer's instructions (ABI PRISM 7000 Sequence Detection System; Applied Biosystems, Rotkreuz, Switzerland). ${ }^{10}$ The following SNPs were analyzed: CYP2D6: allele *3, *4, *5, *6; CYP $3 A 4 * 1 B$ (rs776746), CYP3A4 rs4646437, CYP3A5*3 (rs776746), $C Y P 3 A 7 * 1 C$ (defined by the $-262 T>A,-270 T>G$ ), $A B C B 1$ : $3435 C>T$ (rs1045642), $2677 G>T$ (rs2032582), 1236C>T (rs1128503); NR1/2: rs1523130, rs2472677, and rs7643645. The CYP2D6 *XN gene duplication was analyzed by long PCR. Patients were classified as CYP2D6 poor (PM), intermediate (IM), extensive (EM), or ultrarapid (UM) metabolizer according to the number of functional alleles or gene duplication (www.cypalleles.ki.se). All reagents were purchased from
Applied Biosystems. Internal quality control samples of known genotype were included in all analyses.

\section{Statistical Analysis}

Pharmacokinetics Variability and Associated Factors

The nonparametric Kruskal-Wallis and Wilcoxon/MannWhitney rank tests were used to assess at each time point the association between RIS, OHRIS, the active moiety plasma levels normalized by the dose (hereafter called dose-adjusted plasma levels), and RIS/OHRIS ratio with the studied genotypes and sex, whereas Spearman rank correlation coefficient $\left(r_{\mathrm{s}}\right)$ were used to analyze their association with age.

\section{Pharmacodynamics Variability and Associated Factors}

The association of CGI and SAS scores, weight gain, and CHOLtot, HDL, and prolactin levels with (i) the drug plasma levels, (ii) age, and (iii) RIS doses were assessed by Spearman rank correlation coefficient and with (iv) sex and (v) selected 


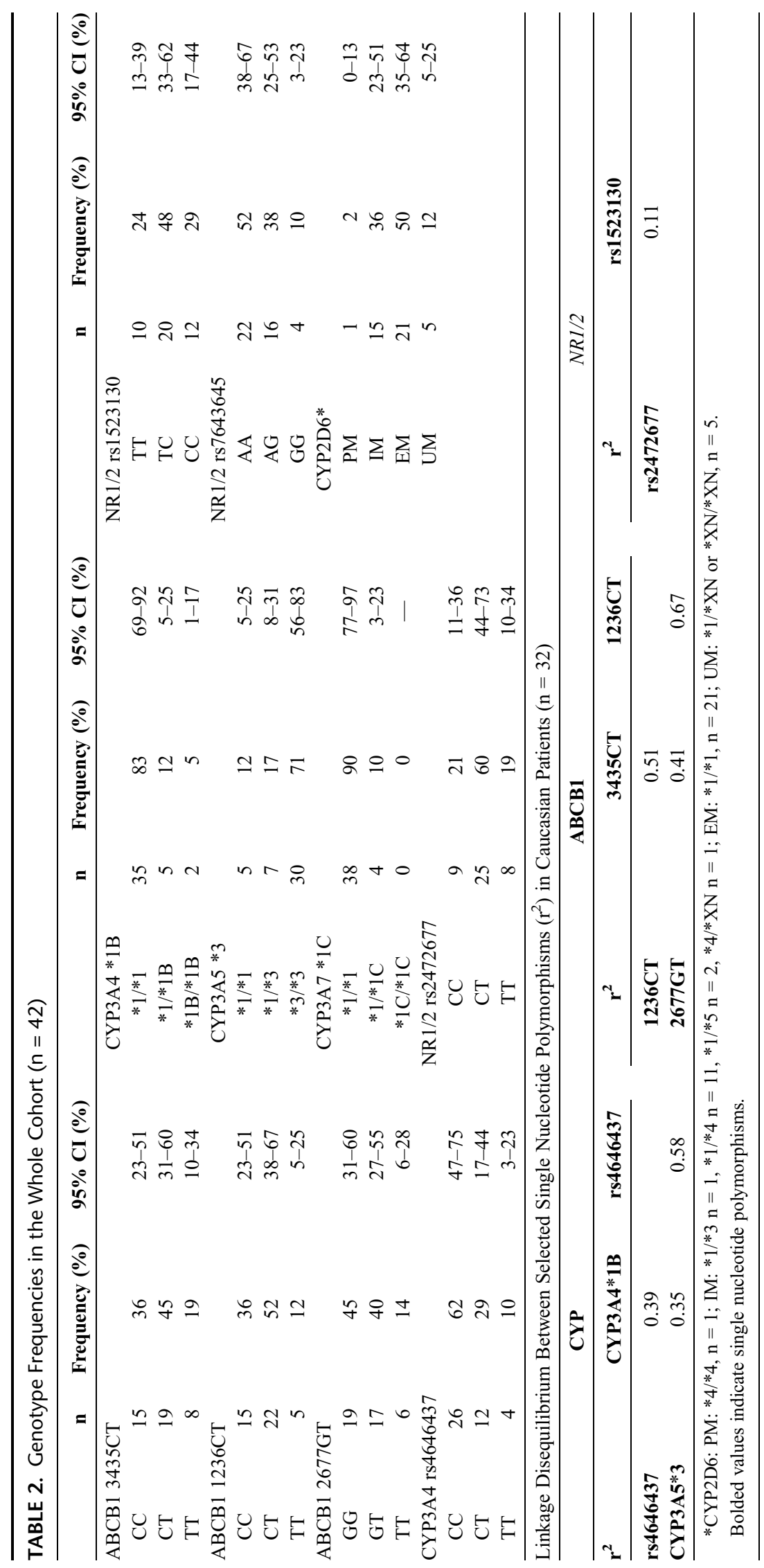


SNPs by Kruskal-Wallis and Wilcoxon/Mann-Whitney rank tests. Incidences of extrapyramidal adverse effect, dyslipidemia, and of elevated prolactin plasma levels were compared between genotypes and clinical variables by Pearson $\chi^{2}$ test.

To take into account the 2 time points, a mixed-effect linear regression adjusted for age and sex was performed, and the validity of the model was assessed by graphical residual diagnostics. Measurements are given as median and interquartile range (IQR) or mean (SD) for results obtained from the fitted mixed model, unless otherwise indicated. The effect sizes were calculated at day 0 using Cohen $d$, which is the difference between means divided by the pooled SD. In case of more than 2 groups, the double mutated versus the wild-type genotype or the CYP2D6 IM versus the UM genotype was considered.

Concomitant drugs that could modify the studied parameters were taken into account, including strong or moderate inhibitors of CYP3A and CYP2D6, CYP3A inducers, drugs causing weight gain, lipid-lowering agents, and anticholinergic agents, ${ }^{26}$ as well as relevant clinical variables (ie, age, sex). Patients receiving a strong CYP2D6 inhibitor were defined as CYP2D6 PM. The allele frequencies are reported with their $95 \%$ confidence interval $(95 \% \mathrm{CI})$ calculated by the adjusted Wald method. The SNPs were tested for Hardy-Weinberg equilibrium using the genhwi command of Stata. Linkage disequilibrium was calculated using $r^{2}$ parameter. The statistical analyses were performed using Stata 11 (StataCorp, College Station, TX). All tests were 2 -sided, and $P \leq 0.05$ was considered statistically significant.

\section{RESULTS}

\section{Patient Characteristics}

Table 1 shows patient clinical characteristics. Sixty-seven patients receiving RLAI were screened for inclusion (15 refusals to participate, 10 not meeting all inclusion criteria). The present results are based on 42 patients. Because of a possible intake of an oral dose of RIS at day 0 , data for 1 patient were considered only at day 7 . The second independent group originally included 28 RLAI patients, among them 16 patients met the inclusion criteria and were incorporated to test result replication (Table 1, replication group). No data were available on SAS score and prolactin levels in the replication group.

\section{Genotype Frequencies}

Genotype frequencies and Caucasian linkage disequilibrium are presented in Table 2 . The observed allele frequencies in Caucasians $(n=32)$ are in line with reported results. ${ }^{14,27}$ All studied SNPs were in Hardy-Weinberg equilibrium $(P>0.05)$. Arbitrarily, 1 patient with the $C Y P 2 D 6 * 4 / * X N$ genotype was classified as an IM. No associations were found for the haplotypes of $A B C B 1, C Y P 3 A$, and NR1/2 with RIS exposure or clinical responses (data not shown).

\section{Pharmacokinetic Variability and Associated Factors}

\section{Plasma Concentration}

Five patients received one of the following CYP2D6 inhibitors: weak inhibitors (citalopram $n=1$, escitalopram $n=2$ ) and strong inhibitors (paroxetine $\mathrm{n}=1$ and levomepromazine $\mathrm{n}=1$ ). The strong CYP3A inhibitor grapefruit juice was taken by 2 (day 0 ) and 1 patient (day 7), respectively. No CYP3A inducers were prescribed. Table 3 shows the median (IQR) RIS, OHRIS, and active moiety plasma concentrations. Risperidone doses were significantly correlated with plasma levels of RIS, OHRIS, and the active moiety, both at days 0 and 7 (data not shown), as previously reported. ${ }^{17,28}$ The dose-adjusted plasma concentrations displayed large interindividual variability (37-, 38-, and 12-fold for RIS, OHRIS, and active moiety, respectively at day $0 ; 18-, 10-$, and 6-fold, respectively, at day 7).

\section{Influence of $C Y P 2 D 6$ and $C Y P 3 A$ Genotypes}

Because only 1 patient was genotyped as a CYP2D6 PM, the statistical analyses were first performed with IM for reference. The CYP2D6 genotypes significantly influenced the doseadjusted RIS plasma levels (test for trend, day 0: $P=0.023$, Cohen $d=1.03$; day 7: $P=0.031$; Fig. 1 ), with dose-, age-, and sex-adjusted RIS plasma concentration in UMs being $45 \%$ of IMs values $(P=0.007)$. The RIS/OHRIS ratios were gradually decreased with increasing CYP2D6 activity (median [IQR]: 0.25 [0.17-0.29], 0.33 [0.25-0.50], 0.40 [0.27-0.56], and 6.26 in the UMs, EMs, IMs, and PM, respectively). Thus, the RIS/ OHRIS ratios adjusted for age and sex was for UMs $36 \%$ of IMs' values ( $P=0.006$, Cohen $d=1.00)$. Interestingly, higher RIS/OHRIS ratios were measured in the present study with RLAI than in a previous study with oral RIS, ${ }^{29}$ supporting the idea that RIS injections may be associated with higher RIS/ OHRIS ratios than oral RIS. ${ }^{29}$ No influence of CYP2D6 genotype was found on dose-adjusted OHRIS $(P>0.7)$ or active moiety $(P>0.1)$.

Then, taken both the lone PM with the 2 patients receiving strong CYP2D6 inhibitors as potential CYP2D6 PM, a 3.3fold higher dose-corrected RIS level $(P=0.001)$, 2.7-fold lower dose-corrected OHRIS level $(P<0.001)$, and 9.1-fold higher RIS/OHRIS ratio $(P<0.001)$ were found as compared with the other CYP2D6 combined genotypes. No association was found with the dose-corrected active moiety plasma levels (1.1-fold, $P>0.1)$. On the other hand, CYP3A SNPs, CYP3A activity determined by the midazolam phenotyping test, CYP3A strong inhibitor comedications $(\mathrm{n}=3)$ were found not to be associated with RIS, OHRIS, and active moiety plasma levels; RIS/ OHRIS ratios; or dose requirements (data not shown). The CYP3A activity measured by the midazolam phenotyping test was not associated with $C Y P 3 A$ and $N R 1 / 2$ SNPs (data not shown).

\section{Influence of $A B C B 1$ and $N R 1 / 2$ Genotypes}

No association was found between $A B C B 1$ SNPs and doseadjusted plasma levels of RIS, OHRIS, active moiety, and RIS/ OHRIS ratios or RLAI dose requirement $(P>0.1)$. On the other hand, and interestingly, a significant association was found at baseline for $N R 1 / 2$ rs $7643645 A>G$, with a 2.6-fold lower doseadjusted of OHRIS $(P=0.033$, Cohen $d=0.99)$ and 2.8-fold lower active moiety $(P=0.031$, Cohen $d=1.18)$ in $G G$ compared with the $A A$ genotype (Table 3 ). The novelty of this finding was assessed in a second independent cohort $(\mathrm{n}=16$, Table 1). In line with the previous results, 2.5 -fold lower doseadjusted OHRIS $(P=0.027$, Cohen $d=2.30)$ and active moiety $(P=0.015$, Cohen $d=1.96)$ plasma levels were found with the $G G$ genotype compared with $A A$ carriers. In addition, similar results were obtained when the 2 cohorts were combined (n $=57$, OHR $P=0.018$, active moiety $P=0.001$ ).

\section{Pharmacodynamics Variability and Associated Factors}

\section{Clinical Response (CGI) and Extrapyramidal Adverse Effects (SAS)}

The median baseline CGI score was 3 points (IQR, 2-4 points), which corresponded to a mild psychiatric illness. Patients 
TABLE 3. RIS, OHRIS, and Active Moiety (RIS + OHRIS) Plasma Levels

\begin{tabular}{|c|c|c|c|c|c|c|c|c|c|}
\hline & \multirow[b]{2}{*}{$\mathbf{n}$} & & \multirow[b]{2}{*}{$\%$} & \multicolumn{2}{|c|}{ RIS } & \multicolumn{2}{|c|}{ OHRIS } & \multicolumn{2}{|c|}{ Active Moiety } \\
\hline & & & & Median & IQR & Median & IQR & Median & IQR \\
\hline \multicolumn{10}{|l|}{ Dose } \\
\hline \multicolumn{10}{|c|}{ Levels at day 0} \\
\hline All & 41 & & & 5 & $3-9$ & 12 & $8-18$ & 21 & $12-27$ \\
\hline $25 \mathrm{mg}$ & 12 & & 29 & 3 & $2-8$ & 9.5 & $5-13$ & 12.5 & $8-24$ \\
\hline $37.5 \mathrm{mg}$ & 9 & & 22 & 4 & $4-6$ & 16 & $12-17$ & 21 & $16-24$ \\
\hline $50 \mathrm{mg}$ & 16 & & 39 & 5.5 & $3-19$ & 14.5 & $9-18$ & 23.5 & $14-38$ \\
\hline $75 \mathrm{mg}$ & 4 & & 10 & 6.5 & $4-10$ & 18.5 & $9-31$ & 27.5 & $17-38$ \\
\hline \multicolumn{10}{|c|}{ Levels at day $7 *$} \\
\hline All & 42 & & & 5 & $3-8$ & 15.5 & $9-21$ & 21 & $14-28$ \\
\hline $25 \mathrm{mg}$ & 13 & & 31 & 3 & $2-6$ & 9 & $8-13$ & 12 & $10-21$ \\
\hline $37.5 \mathrm{mg}$ & 9 & & 21 & 6 & $3-8$ & 18 & $11-20$ & 24 & $18-28$ \\
\hline $50 \mathrm{mg}$ & 16 & & 38 & 5.5 & $5-11$ & 16.5 & $11-26$ & 23.5 & $19-40$ \\
\hline \multirow[t]{3}{*}{$75 \mathrm{mg}$} & 4 & & 10 & 5 & $4-12$ & 23 & $20-25$ & 29.5 & $27-35$ \\
\hline & & & & \multicolumn{2}{|c|}{ RIS } & \multicolumn{2}{|c|}{ OHRIS } & \multicolumn{2}{|c|}{ Active Moiety } \\
\hline & \multicolumn{3}{|c|}{$\mathbf{n}$} & Median & IQR & Median & IQR & Median & IQR \\
\hline \multicolumn{10}{|l|}{ Genotype } \\
\hline \multicolumn{10}{|c|}{$N R 1 / 2$ rs7643645 } \\
\hline $\mathrm{AA}$ & & 22 & & 0.12 & $0.08-0.20$ & 0.34 & $0.21-0.45$ & 0.51 & $0.32-0.64$ \\
\hline $\mathrm{AG}$ & & 15 & & 0.12 & $0.10-0.36$ & 0.38 & $0.26-0.48$ & 0.56 & $0.36-0.96$ \\
\hline GG & & 4 & & 0.07 & $0.05-0.10$ & 0.13 & $0.08-0.23$ & 0.18 & $0.16-0.30$ \\
\hline$P_{\text {(Kruskal-Wall }}$ & & & & 0.130 & & 0.033 & & 0.030 & \\
\hline
\end{tabular}

Median and IQR are shown. Top: At day of injection (day 0) and at day 7 according to the fortnightly dose of RLAI (ng/mL). Bottom: At day 0 according to the $N R 1 / 2$ rs 7643645 genotypes for dose-adjusted plasma levels (ng/mL.mg).

*One additional patient at day 7 excluded from day 0 because of a suspicion of oral RIS intake.



FIGURE 1. Plasma concentrations corrected by the dose (ng/mL·mg) of RIS, OHRIS, and the active moiety (RIS + OHRIS) and RIS/OHRIS ratio adjusted by age and sex for CYP2D6 genotype. Data were obtained according to the mixed model and showed as mean \pm SE. ${ }^{*} P<0.01$. Mean (SE) of RIS/OHRIS ratio adjusted by age and sex according by CYP2D6 genotypes: PM 6.43, IM 0.70 (0.14), EM $0.46(0.12)$, UM $0.16(0.24)$. 
had very variable duration of treatment, and expectedly, no significant correlations were found between CGI scores and fortnightly dose $(P>0.9)$, dose-adjusted RIS $(P>0.1)$, OHRIS $(P>0.7)$, and active moiety $(P>0.3)$ plasma levels at baseline, at day 7 , and using a mixed model (data not shown). No associations were found between CGI scores and other clinical demographic or genetic data (data not shown).

The median baseline SAS score was 3 points (IQR, 1-5 points), and $55 \%$ had SAS scores greater than 3 or were receiving an anticholinergic agent (biperiden, $\mathrm{n}=6$ among whom 3 had still an SAS score $>3$ ). NR1/2 rs2472677CC carriers had higher SAS score $(\mathrm{n}=9$; median, 5; IQR, 4-7) than the $C T$ and $T T$ carriers $(\mathrm{n}=33$; median, 2 ; IQR, $0-5)$ at day 0 and at day 7 (Cohen $d=0.78, P \leq 0.03$ on both cases), also in the mixed model $(P=0.035)$. Although a tendency was observed in the subgroup without anticholinergic agent $(\mathrm{n}=7$ [median, 4; IQR, 3-11], $\mathrm{n}=29$ [median, 2; IQR, 0-5] for CC vs CT and TT genotypes, respectively), no significant association was found at days 0 and $7(P=0.1$ in each case, $P=0.08$ in the mixed model). Finally, no associations were found between SAS scores and RLAI dose; treatment length; RIS, OHRIS, and active moiety plasma levels; studied genotypes; or with CYP3A activity (data not shown).

\section{Weight Gain and Lipid Profile}

High prevalence of several cardiovascular risk factors was observed in the present cohort, with $26 \%$ of obesity, $81 \%$ smokers, and $45 \%$ patients presenting a dyslipidemia. A median weight gain of $6 \mathrm{~kg}$ (IQR, $0-16 \mathrm{~kg}$ ) for a median duration of RLAI treatment of 8 months (IQR, 4-15 months) was found for the 39 patients with available data. It is noteworthy to mention that 10 patients received other drugs potentially linked to weight gain (valproate $n=4$, clozapine $n=1$, mirtazapine $n=2$, quetiapine $\mathrm{n}=2$, mirtazapine and quetiapine in combination $\mathrm{n}=1$ ), and 4 patients had lipid-lowering agents. Weight gain and CHOLtot and HDL levels were not correlated to fortnightly dose; RIS, OHRIS, and active moiety dose-adjusted plasma levels; or selected SNPs in the whole population and in the subgroups without weight gain-related drug or lipid-lowering agents $(P>0.1)$.

Sex difference in HDL levels was observed as expected (median [IQR] HDL levels in women: $1.65[1.23-2.05] \mathrm{mmol} / \mathrm{L}$; in men: 1.11 [0.95-1.38] mmol/L, $P=0.004$ ). Interestingly, HDL levels were significantly correlated to CYP3A activity in the whole population $\left(r_{\mathrm{s}}=0.49, P=0.001\right)$. Thus, median (IQR) HDL levels of $0.95(0.9-1) \mathrm{mmol} / \mathrm{L}(\mathrm{n}=5)$ and $1.19(0.98-1.46)$ $\mathrm{mmol} / \mathrm{L}(\mathrm{n}=22)$ were measured in men with low CYP3A activity $(<1.9 \mathrm{MR})$ and with an extensive activity $(\geq 1.9 \mathrm{MR} ; P=$ 0.029 , Cohen $d=1.20$ ), respectively. Lower HDL level was observed in the lone woman with low CYP3A activity than those with extensive CYP3A activity: $1.1 \mathrm{mmol} / \mathrm{L}(\mathrm{n}=1)$ and 1.68 $(1.4-2.05) \mathrm{mmol} / \mathrm{L}(\mathrm{n}=10)$, respectively. CYP3A activity adjusted for age and sex remained significantly associated with HDL levels in the whole population $(P=0.014)$ and in the subgroup without lipid-lowering agent $(P=0.029)$.

\section{Prolactin Plasma Concentration}

Sex difference in prolactin levels was found as expected (day 0 median [IQR] prolactin level: $\mathrm{n}=30,27(19-45) \mathrm{ng} / \mathrm{mL}$ in men; $\mathrm{n}=12,75(46-133) \mathrm{ng} / \mathrm{mL}$ in women; $P<0.001)$. High prolactin levels were more frequent in women $(75 \%$ of women, and $27 \%$ of men; $x^{2}, P=0.004$ ), as previously reported, ${ }^{23,30}$ and were measured in $40 \%$ and $45 \%$ of patients at day 0 and at day 7 , respectively. Hyperprolactinemia symptoms were reported by 6 patients ( 3 women, 3 men; sexual dysfunction, $\mathrm{n}=3$; galactorrhea, $\mathrm{n}=1$; gynecomastia, $\mathrm{n}=1$; and amenorrhea, $\mathrm{n}=1$ ), which were not associated with particularly high prolactin levels (median, 37 [20-55] ng/mL).

Prolactin levels were significantly correlated with RIS, OHRIS, and active moiety plasma levels $\left(r_{\mathrm{s}}=0.43, P=0.0047\right.$; $r_{\mathrm{s}}=0.33, P=0.037 ; r_{\mathrm{s}}=0.38, P=0.013$, respectively) at day 0 . Significant association was found between RIS exposure and prolactin levels using a mixed model adjusted for age and sex (RIS $P=0.001$, OHRIS $P<0.001$, and active moiety $P<$ 0.001 ). Of note, $47 \%$ of the variation of prolactin level can be explained by the model including age, sex, and active moiety plasma level. Fortnightly dose was not found to be associated with prolactin levels $(P=0.47)$. Prolactin levels adjusted for age and sex were significantly associated with $N R 1 / 2$ $r s 2472677 C>T$ polymorphism $(P=0.032)$. At day $0,1.8$-fold (in women, Cohen $d=0.73$ ) and 1.3-fold (in men, Cohen $d=$ 0.18 ) lower prolactin levels were found in carriers of the $C C$ genotypes as compared with the $C T$ and $T T$ genotypes.

\section{DISCUSSION}

This study aimed to analyze, in patients with RLAI treatment, the influence of gene polymorphisms potentially linked to RIS pharmacokinetics and of the CYP3A activity on the plasma levels of RIS, OHRIS, and active moiety and on potential drug-related adverse effects.

\section{Pharmacokinetic Variability and Associated Factors}

\section{Influence of CYP2D6 and CYP3A Genotypes}

A very large interindividual variability was measured for dose-adjusted plasma concentrations of RIS, OHRIS, and active moiety in patients receiving RLAI, in agreement with a previous report. ${ }^{17}$ CYP2D6 activity was found to be a major determinant of RIS pharmacokinetics. The CYP2D6 genotype showed a significant influence on dose-adjusted plasma levels of RIS and RIS/OHRIS ratio but not on dose-adjusted OHRIS and active moiety, similar to results with oral RIS. ${ }^{11,31}$ It is possible that different levels of CYP2D6 activity may not have major effects on the total moiety elimination unless the CYP2D6 activity is absent, as shown in our and other results, ${ }^{31}$ although discrepancies have been published. ${ }^{9}$ In addition, we confirmed the influence of strong CYP2D6 inhibitors on RIS metabolism. ${ }^{3,4}$

Although not statistically significant, CYP2D6 PMs seemed to be underrepresented with only 1 (3\%; $95 \%$ CI, $0 \%-17 \%)$ patient with this genotype of 32 Caucasians as compared with the $5 \%$ to $10 \%$ frequency expected in the Caucasian population. ${ }^{29}$ In contrast, CYP2D6 UMs seemed to be overrepresented in the present study (5 patients, $12 \%$ [95\% CI, 6\%-32\%), compared with the $3 \%$ to $5 \%$ reported in Caucasian populations. ${ }^{29}$ Therefore, the possibility of an earlier treatment discontinuation in PM patients due to potential adverse effects is questioned. The PM phenotype was previously reported to increase the risk for adverse effects and drug discontinuation in patients receiving oral RIS. ${ }^{19}$ On the other hand, the higher than expected prevalence of UM in the present study raises the question whether patients receiving antipsychotics metabolized mainly or partially by this enzyme (eg, aripiprazole or haloperidol and to a lesser extent quetiapine or olanzapine) might respond inadequately and then be switched to an injection formulation because of suspicion of poor compliance.

In patients receiving oral RIS, the administration of strong CYP3A inhibitors or inducers resulted in high or low plasma levels of RIS, OHRIS, and of the active moiety, respectively. ${ }^{5,7}$ 
The present study on RIS depot formulation showed no association between CYP3A activity with dose-adjusted RIS, OHRIS, and active moiety plasma levels. As the midazolam phenotype test was suggested to reflect CYP3A activity both in the liver and in the intestines, ${ }^{25}$ one may hypothesize that the depot formulation, by shunting the first-pass effect, reduces the influence of CYP3A in the pharmacokinetics of injected RIS. In agreement with the direct determination of CYP3A activity by the midazolam test, no associations were found between the analyzed CYP3A SNPs and RIS pharmacokinetics. These results are in line with other reports, ${ }^{31,32}$ whereas another study found higher OHRIS and active moiety plasma levels for $C Y P 3 A 5 * 3 * 3 \mathrm{com}-$ pared with the other genotypes in patients receiving oral RIS. ${ }^{8}$

\section{Influence of $A B C B 1$ and $N R 1 / 2$ Genotypes}

It has recently been shown that the $A B C B 1$ SNPs do not influence or only moderately influence the steady-state plasma concentrations of RIS and OHRIS after the oral intake of RIS. ${ }^{9,11,31,32}$ In the present study with RIS depot formulation, we showed that $A B C B 1$ SNPs do not significantly contribute to variability of RIS and OHRIS in the blood.

Interestingly, we reported a significant association between NR1/2 SNPs and RIS pharmacokinetics. The rs 1523130, $r s 2472677$, and $r s 7643645$ SNPs were previously identified in transcription binding sites of $N R 1 / 2$ regulatory regions. ${ }^{15}$ In patients with HIV treated with $400 \mathrm{mg} / \mathrm{d}$ of atazanavir, a P-gp and CYP3A substrate, the $r s 2472677 T$ allele is associated with reduced trough plasma concentration of this drug, ${ }^{14}$ which is in line with its reported increased activity. ${ }^{15}$ In the present study, the $N R 1 / 2$ rs $7643645 G$ allele, linked to a decreased transcription, ${ }^{15}$ was found to be associated with lower dose-adjusted OHRIS and active moiety plasma levels. Moreover, these results were replicated in a second independent cohort of patients receiving RLAI as well as when the 2 cohorts were combined. These results could be tentatively explained by the lower $C Y P 3 A$ and $A B C B 1$ transcription activity measured in subjects with the $G$ allele. ${ }^{15}$ However, because CYP3A activity measured by the midazolam phenotyping test was not found to be associated with RIS pharmacokinetics, the influence of PxR observed in the present study could not be explained by the induction of CYP3A but rather by the induction of other drug metabolizing enzymes, transporters, and/or P-gp. The influence of $N R 1 / 2$ gene remains, however, to be demonstrated after an oral intake of this drug, due to the first-pass effect and the involvement of other confounding factors.

\section{Pharmacodynamic Variability and Associated Factors}

\section{Clinical Response (CGI) and Extrapyramidal Adverse Effects (SAS)}

In line with previous reports, no significant correlation was found between RIS, OHRIS, and active moiety plasma levels, dose and CGI score, ${ }^{4,33}$ or SAS scores. ${ }^{1}$ The criteria of 4 injections might have discarded nonresponders with high CGI score, whereas the long treatment duration (median of 8.5 months) might contribute to the overall low CGI score in the group (median score of 3 ).

Because of conflicting results, it remains unclear whether EPSs are linked to RIS dose or plasma concentrations, ${ }^{1,3,5,19}$ although $34 \%$ of patients with RLAI have EPSs, making these adverse effects an important clinical issue. ${ }^{34}$ CYP2D6 PMs were suggested to be at higher risk for tardive dyskinesia and RIS discontinuation due to adverse drug reactions. ${ }^{19}$ In the present study, as there was only 1 CYP2D6 PM, no conclusion can be drawn on this point. When taking into account the 3 patients with a presumably CYP2D6 PM status, no association was observed for the drug adverse effects. We did not find any influence of $C Y P 3 A$ genotypes and SAS scores, which is in agreement with the lack of association between $C Y P 3 A$ genotypes and plasma levels and reports showing that association of EPSs and strong CYP3A inhibitors remains unclear. ${ }^{5,32}$ Our result might also support the idea that RLAI may be associated with higher RIS/OHRIS ratios than oral RIS. ${ }^{19}$ Its clinical consequences require further investigation as an increased ratio was related to more adverse effects, ${ }^{19}$ whereas other reports indicated less EPSs with RLAI than with oral RIS. ${ }^{16,34}$

Because of its strategic localization at the blood-brain barrier, P-gp was hypothesized to influence EPSs. A previous study with oral RIS found that $A B C B 1$ 2677T-3435T carriers presented an increased occurrence of EPSs, which were not linked to plasma concentrations. ${ }^{9}$ No such associations were found in the present study, which differ by many variables (oral form, drug-naive patients, short-term study with 8 weeks of follow-up, and a majority of women in the former study). Interestingly, we found a higher SAS score for NR1/2 rs2472677CC genotype, which was reported to be linked to a decreased activity, ${ }^{15}$ as compared with the $T$ carriers. Thus, one could hypothesize that decreased activity of P-gp and/or of other transporters at the blood-brain barrier could increase RIS and OHRIS brain levels and therefore explain the observed increase of EPSs. This result should be assessed in a bigger cohort as the association was anymore significant when patients with anticholinergic agents ( 6 of the 42 patients) were excluded.

\section{Weight Gain and Metabolic Profile}

In total, $38 \%$ of patients gained more than $10 \%$ of their baseline weight (median weight gain was $4.5 \mathrm{~kg}$ for a median treatment duration of 8.5 months), and $45 \%$ of patients presented dyslipidemia, the latter being not a frequent adverse effect related to oral RIS treatment or to RLAI ${ }^{26}$ However, RIS could indirectly influence the lipid pathway through the increased risk of weight gain during long-term treatment. This is in agreement with results showing lipid parameter impairment during long-term RIS monotherapy in an Asian population. ${ }^{35}$ Concerning HDL levels, the significant positive correlation found in the present study between CYP3A activity and higher HDL levels is in accordance with similar findings in a rat model. An increased HDL level was observed in rats treated with inducers of CYP3A activity as compared with controls, and this increase was proportional to the potency of each CYP3A inducer. ${ }^{36}$ Moreover, the PxR inducer rifampin was reported to increase HDL levels in mice but not in PxR-knockout mice. ${ }^{36}$ Similar effect is also suggested in humans: patients receiving anticonvulsant (ie, phenobarbital, phenytoin, two CYP3A indu$\operatorname{cers}^{26}$ ) showed increased HDL levels as compared with healthy matched controls. ${ }^{37}$ A significant correlation was also found between HDL levels and the corresponding CYP content in 18 human livers obtained by percutaneous biopsy, with CYP3A being the major CYP enzyme in the liver. ${ }^{37}$

\section{Prolactin Plasma Concentration}

High prevalence of elevated prolactin levels $(43 \%$ of patient) was found, which is in accordance with reports for $\mathrm{RLAI}^{38}$ and for oral RIS, ${ }^{30}$ although the adverse effect intensity for RLAI compared with oral RIS is still not clearly demonstrated. ${ }^{39}$ Interestingly, a significant positive correlation was found between prolactin levels and RIS, OHRIS, and active 
moiety plasma levels. Prolactin level adjusted for age and sex was also significantly associated with $N R 1 / 2$ rs $2472677 C>T$ polymorphism, but with lower levels in carriers of the $C C$ genotypes as compared with the $C T$ and $T T$ genotypes. No explanation can be presently proposed to explain the apparent discrepancy that the same genotypes (ie, CC) are associated with increased EPSs and decreased prolactin levels. One can, however, mention that occurrence of EPSs is thought to be mediated by $\mathrm{D}_{2}$ receptor antagonism at the level of the striatum, whereas the release of prolactin is induced by $\mathrm{D}_{2}$ blockade at the level of the pituitary. ${ }^{30,40}$ Furthermore, whereas the striatum is located in the central nervous system part protected by the blood-brain barrier, the pituitary is outside this barrier. ${ }^{40}$ Thus, as NR1/2 SNP affects the activities of both metabolic enzymes and transporters, the same SNP could affect differently the drugs activity, depending on the location of the target organ.

\section{Limitations}

This study has several limitations: first, its naturalistic crosssectional design, which leads to a heterogeneous RLAI medication including different treatment durations, variable doses, and psychiatric diagnostics. It excludes to draw any conclusion of RLAI consequences on clinical parameters (ie, prolactin and cholesterol levels, EPSs), as baseline parameters are unknown, and preexisting morbid status could not be rejected. Thus, only associations are presented here. This design, in addition to the fact that the CGI score is a very simple scale, limits the value of our data on therapeutic response as well as the statistical analysis performed on these data. On the other hand, this limitation should not apply to the blood levels of the RIS, OHRIS, and active moiety. Second, dyslipidemia was defined without triglyceride level indications as this parameter was not available. The last limitation is the modest number of patients included and the statistical analysis, which were not corrected for the multiple tests performed. Thus, the new findings of the present study must be considered as preliminary and must be validated in other cohorts, although the significant associations between NR1/2 SNP and RIS, OHRIS, and active moiety have already been replicated in a second data set of patients receiving RLAI. On the other hand, the strength of this study on depot formulation is that, by definition, the compliance of the patients is ascertained, which is important considering the poor compliance observed with oral formulation on long-term treatment. $^{34}$

\section{CONCLUSIONS}

In agreement with previous reports on oral RIS, CYP2D6 was also found to significantly contribute to RIS pharmacokinetics in patients treated with RLAI. CYP3A activity measured by a midazolam phenotyping test, and $C Y P 3 A$ and $A B C B 1$ SNPs were not found to be associated with RIS, OHRIS, or the active moiety plasma levels. Accordingly, adverse effects induced by RIS may not be associated with these SNPs. Prolactin levels were found to be related to RIS exposure. Interestingly, NRI/2 SNPs were significantly associated with RIS, OHRIS, and active moiety plasma levels, with the extrapyramidal adverse effects, and with the prolactin levels. These results must be replicated in other population with RLAI, and it should also be determined whether it is also relevant for the oral form of RIS.

\section{ACKNOWLEDGMENTS}

The authors thank C. Brogli (logistical assistance), E. Ponce (bibliographical help), Drs S. Crettol and L. Quteineh (scientific discussion); M. Brocard, N. Cochard, M. Delessert, K. Powell Golay, A. C. Aubert, A. Kottelat, M. Brawand, V. Hodel, and S. Jacquet (sample analysis); Dr M. Gholam-Rezaee (statistical help); and Drs B. Knezevic, N. Lababidi, P. Stephan, P. Morena, and A. Meiler (recruitment and clinical investigations).

\section{AUTHOR DISCLOSURE INFORMATION}

Dr Bondolfi is a consultant of Pfizer, received grants from Janssen-Cilag, and received speaker fees paid for by many different pharmaceutical societies, medical societies, hospitals, and congress organizations. Dr Conus received a nonrestricted educational grant from Bristol-Myers Squibb and JanssenCilag and supports for attending conferences from Eli Lilly and Bristol-Myers Squibb. Dr Eap received nonrestricted educational grants from Janssen-Cilag and Bristol-Myers Squibb. He received honoraria for conferences or teaching CME courses from AstraZeneca, Bristol-Myers Squibb, Eli Lily, Essex Chemie, GlaxoSmithKline, Janssen-Cilag, Lundbeck, Novo Nordisk, and Organon. Ms Jaquenoud Sirot was in the advisory board of Janssen-Cilag and received speaker fees paid for by many different pharmaceutical societies, medical societies, hospitals, and congress organizations. The other authors declare no conflicts of interest.

\section{REFERENCES}

1. Spina E, Avenoso A, Facciola G, et al. Relationship between plasma risperidone and 9-hydroxyrisperidone concentrations and clinical response in patients with schizophrenia. Psychopharmacology (Berl). 2001;153:238-243.

2. Kleinberg DL, Davis JM, de Coster R, et al. Prolactin levels and adverse events in patients treated with risperidone. J Clin Psychopharmacol. 1999;19:57-61.

3. Bondolfi G, Eap CB, Bertschy G, et al. The effect of fluoxetine on the pharmacokinetics and safety of risperidone in psychotic patients. Pharmacopsychiatry. 2002;35:50-56.

4. Spina E, Avenoso A, Facciola G, et al. Plasma concentrations of risperidone and 9-hydroxyrisperidone during combined treatment with paroxetine. Ther Drug Monit. 2001;23:223-227.

5. Jung SM, Kim KA, Cho HK, et al. Cytochrome P450 3A inhibitor itraconazole affects plasma concentrations of risperidone and 9-hydroxyrisperidone in schizophrenic patients. Clin Pharmacol Ther. 2005;78:520-528.

6. Spina E, Avenoso A, Facciola G, et al. Plasma concentrations of risperidone and 9-hydroxyrisperidone: effect of comedication with carbamazepine or valproate. Ther Drug Monit. 2000;22:481-485.

7. Kim KA, Park PW, Liu KH, et al. Effect of rifampin, an inducer of CYP3A and P-glycoprotein, on the pharmacokinetics of risperidone. $J$ Clin Pharmacol. 2008;48:66-72.

8. Kang RH, Jung SM, Kim KA, et al. Effects of CYP2D6 and CYP3A5 genotypes on the plasma concentrations of risperidone and 9-hydroxyrisperidone in Korean schizophrenic patients. J Clin Psychopharmacol. 2009;29:272-277.

9. Jovanovic N, Bozina N, Lovric M, et al. The role of CYP2D6 and $\mathrm{ABCB} 1$ pharmacogenetics in drug-naive patients with first-episode schizophrenia treated with risperidone. Eur J Clin Pharmacol. 2010;66:1109-1117.

10. Eap CB, Buclin T, Hustert E, et al. Pharmacokinetics of midazolam in CYP3A4- and CYP3A5-genotyped subjects. Eur J Clin Pharmacol. 2004;60:231-236.

11. Yasui-Furukori N, Mihara K, Kondo T, et al. Effects of CYP2D6 genotypes on plasma concentrations of risperidone and enantiomers of 9-hydroxyrisperidone in Japanese patients with schizophrenia. J Clin Pharmacol. 2003;43:122-127. 
12. Wang JS, Ruan Y, Taylor RM, et al. The brain entry of risperidone and 9-hydroxyrisperidone is greatly limited by P-glycoprotein. Int J Neuropsychopharmacol. 2004;7:415-419.

13. Kliewer SA, Goodwin B, Willson TM. The nuclear pregnane $\mathrm{X}$ receptor: a key regulator of xenobiotic metabolism. Endocr Rev. 2002;23:687-702.

14. Siccardi M, D'Avolio A, Baietto L, et al. Association of a single-nucleotide polymorphism in the pregnane $\mathrm{X}$ receptor (PXR 63396C->T) with reduced concentrations of unboosted atazanavir. Clin Infect Dis. 2008;47:1222-1225.

15. Lamba J, Lamba V, Strom S, et al. Novel single nucleotide polymorphisms in the promoter and intron 1 of human pregnane $\mathrm{X}$ receptor/NR1I2 and their association with CYP3A4 expression. Drug Metab Dispos. 2008;36:169-181.

16. McEvoy JP. Risks versus benefits of different types of long-acting injectable antipsychotics. J Clin Psychiatry. 2006;67(suppl 5):15-18.

17. Castberg I, Spigset O. Serum concentrations of risperidone and 9-hydroxyrisperidone after administration of the long-acting injectable form of risperidone: evidence from a routine therapeutic drug monitoring service. Ther Drug Monit. 2005;27:103-106.

18. Albrecht A, Morena PG, Baumann P, et al. High dose of depot risperidone in a nonresponder schizophrenic patient. J Clin Psychopharmacol. 2004;24:673-674

19. de Leon J, Susce MT, Pan RM, et al. The CYP2D6 poor metabolizer phenotype may be associated with risperidone adverse drug reactions and discontinuation. J Clin Psychiatry. 2005;66:15-27.

20. Guy W. ECDEU Assessment Manual for Psychopharmacology. Rockville, MD: US Department of Health and Human Services Publication (ADM); 1976:218-222.

21. Hawley C, Fineberg N, Roberts A, et al. The use of the Simpson Angus Scale for the assessment of movement disorder: a training guide. Int J Psychiatr Clin Pract. 2003;7:249-257.

22. Third Report of the National Cholesterol Education Program (NCEP) Expert Panel on Detection, Evaluation, and Treatment of High Blood Cholesterol in Adults (Adult Treatment Panel III) final report. Circulation. 2002;106:3143-3421.

23. Halbreich U, Kahn LS. Hyperprolactinemia and schizophrenia: mechanisms and clinical aspects. J Psychiatr Pract. 2003;9:344-353.

24. Eap CB, Bouchoux G, Powell Golay K, et al. Determination of picogram levels of midazolam, and 1- and 4-hydroxymidazolam in human plasma by gas chromatography-negative chemical ionization-mass spectrometry. J Chromatogr B Analyt Technol Biomed Life Sci. 2004;802:339-345.

25. Eap CB, Buclin T, Cucchia G, et al. Oral administration of a low dose of midazolam (75 microg) as an in vivo probe for CYP3A activity. Eur J Clin Pharmacol. 2004;60:237-246.
26. Morant J, ed. Compendium Suisse de Médicaments. Bâle (Suisse): Documed AG, 2010. Available at: http://www.kompendium.ch. Accessed June 6, 2011.

27. National Center for Biotechnology Information-dbSNP, 2011. Available at: http://www.ncbi.nlm.nih.gov/snp. Accessed June 6, 2011.

28. Eerdekens M, Van Hove I, Remmerie B, et al. Pharmacokinetics and tolerability of long-acting risperidone in schizophrenia. Schizophr Res. 2004;70:91-100.

29. de Leon J, Susce MT, Johnson M, et al. DNA microarray technology in the clinical environment: the AmpliChip CYP450 test for CYP2D6 and CYP2C19 genotyping. CNS Spectr. 2009;14:19-34.

30. Melkersson KI. Prolactin elevation of the antipsychotic risperidone is predominantly related to its 9-hydroxy metabolite. Hum Psychopharmacol. 2006;21:529-532.

31. Gunes A, Spina E, Dahl ML, et al. ABCB1 polymorphisms influence steady-state plasma levels of 9-hydroxyrisperidone and risperidone active moiety. Ther Drug Monit. 2008;30:628-633.

32. Leon J, Susce MT, Pan RM, et al. A study of genetic (CYP2D6 and $\mathrm{ABCB} 1)$ and environmental (drug inhibitors and inducers) variables that may influence plasma risperidone levels. Pharmacopsychiatry. 2007;40:93-102.

33. Kakihara S, Yoshimura R, Shinkai K, et al. Prediction of response to risperidone treatment with respect to plasma concencentrations of risperidone, catecholamine metabolites, and polymorphism of cytochrome P450 2D6. Int Clin Psychopharmacol. 2005;20:71-78.

34. Canas F, Moller HJ. Long-acting atypical injectable antipsychotics in the treatment of schizophrenia: safety and tolerability review. Expert Opin Drug Saf. 2010;9:683-697.

35. Murashita M, Inoue T, Kusumi I, et al. Glucose and lipid metabolism of long-term risperidone monotherapy in patients with schizophrenia. Psychiatry Clin Neurosci. 2007;61:54-58.

36. Bachmann K, Patel H, Batayneh Z, et al. PXR and the regulation of apoA1 and HDL-cholesterol in rodents. Pharmacol Res. 2004;50:237-246

37. Luoma PV, Sotaniemi EA, Pelkonen RO, et al. Plasma high-density lipoprotein cholesterol and hepatic cytochrome P-450 concentrations in epileptics undergoing anticonvulsant treatment. Scand J Clin Lab Invest. 1980;40:163-167.

38. Simpson GM, Mahmoud RA, Lasser RA, et al. A 1-year double-blind study of 2 doses of long-acting risperidone in stable patients with schizophrenia or schizoaffective disorder. J Clin Psychiatry. 2006;67:1194-1203.

39. Chue P, Eerdekens M, Augustyns I, et al. Comparative efficacy and safety of long-acting risperidone and risperidone oral tablets. Eur Neuropsychopharmacol. 2005;15:111-117.

40. Ganong WF. Review of Medical Physiology. 22nd ed. LANGE Basic Science Series. New York: The McGraw-Hill Companies; 2005. Chapters 12, 14, 22. 